\title{
Underutilization of Living Donor Liver Transplantation in the United States: Bias against MELD 20 and Higher
}

\author{
Ryan B. Perumpail ${ }^{1}$, Eric R. Yoo ${ }^{2}$, George Cholankeril ${ }^{3}$, Lupe Hogan $^{1}$, Melodie Deis ${ }^{1}$, \\ Waldo C. Concepcion ${ }^{4}$, C. Andrew Bonham ${ }^{4}$, Zobair M. Younossi ${ }^{5}$, Robert J. Wong ${ }^{6}$ \\ and Aijaz Ahmed*1 \\ ${ }^{1}$ Division of Gastroenterology and Hepatology, Stanford University School of Medicine, Stanford, CA, USA; ${ }^{2}$ Department of \\ Medicine, University of Illinois College of Medicine, Chicago, IL, USA; ${ }^{3}$ Division of Gastroenterology and Hepatology, University of \\ Tennessee Health Sciences Center, Memphis, TN, USA; ${ }^{4}$ Division of Surgery, Division of Abdominal Transplantation, Stanford \\ University School of Medicine, Stanford, CA, USA; ${ }^{5}$ Department of Medicine, Center for Liver Diseases, Inova Fairfax Hospital, \\ Falls Church, VA, USA; ${ }^{6}$ Division of Gastroenterology and Hepatology, Alameda Health System, Highland Hospital Campus,
} Oakland, CA, USA

\begin{abstract}
Background and Aims: Utilization of living donor liver transplantation (LDLT) and its relationship with recipient Model for End-Stage Liver Disease (MELD) needs further evaluation in the United States (U.S.). We evaluated the association between recipient MELD score at the time of surgery and survival following LDLT. Methods: All U.S. adult LDLT recipients with MELD < 25 were evaluated using the 1995-2012 United Network for Organ Sharing registry. Survival following LDLT was stratified into three MELD categories (MELD < 15 vs. MELD 15-19 vs. MELD 20-24) and evaluated using KaplanMeier methods and multivariate Cox proportional hazards models. Results: Overall, 2,258 patients underwent LDLT. Compared to patients with MELD $<15$, overall 5-year survival following LDLT was similar among patients with MELD 15-19 $(80.9 \%$ vs. $80.3 \%, p=0.77)$ and MELD $20-24$ (81.2\% vs. $80.3 \%, p=0.73)$. When compared to patients with MELD < 15 , there was no significant difference in long-term post-LDLT survival among those with MELD 15-19 (HR: 1.11 , 95\% CI: $0.85-1.45, p=0.45)$ and a non-significant trend towards lower survival in patients with MELD 20-24 (HR: $1.28,95 \%$ CI: $0.91-1.81, p=0.16)$. Only $14 \%$ of LDLTs were performed in patients with MELD $20-24$ and the remaining $86 \%$ in patients with MELD $<20$. Conclusion: LDLT is underutilized in patients with MELD 20 and higher.

(c) 2016 The Second Affiliated Hospital of Chongqing Medical University. Published by XIA \& HE Publishing Inc. All rights reserved.
\end{abstract}

Keywords: Living donors; Liver transplantation; Waitlist; Model for End-Stage Liver Disease (MELD).

Abbreviations: BMI, body-mass index; DDLT, deceased donor liver transplantation; DM, diabetes mellitus; HCC, hepatocellular carcinoma; HCV, hepatitis C virus; LDLT, living donor liver transplantation; MELD, Model for End-Stage Liver Disease; UNOS/OPTN, United Network for Organ Sharing/Organ Procurement Transplant Network.

Received: 15 August 2016; Revised: 18 September 2016; Accepted: 19 September 2016

DOI: $10.14218 /$ JCTH.2016.00029.

*Correspondence to: Aijaz Ahmed, Division of Gastroenterology and Hepatology, Stanford University School of Medicine, 750 Welch Road, Suite \#210, Stanford, CA 94304, USA. Tel: +1-650-498-6091, Fax: +1-650-498-5692, E-mail: aijazahmed@stanford.edu
Introduction

Deceased donor liver transplantation (DDLT) has not been able to keep pace with the increasing demand for liver transplantation in the United States (U.S.). In 2013, while 10,479 candidates were added to the liver transplant waiting list, only 5,921 liver transplants were performed in adults. ${ }^{1}$ Despite comparable outcomes between living donor liver transplantation (LDLT) and DDLT, only 211 , or $3.7 \%$, of total U.S. liver transplants were LDLT in 2013.1,2 National trends reveal an aversion to offering LDLT to sicker patients with high Model for End-Stage Liver Disease (MELD) scores. MELD at transplant has been shown to be significantly lower among LDLT recipients compared to DDLT recipients. ${ }^{2-4}$ However, it is unclear whether high MELD scores are even associated with lower survival following LDLT. Although the MELD score predicts liver transplantation waitlist survival, ${ }^{5,6}$ there is uncertainty surrounding what upper limit MELD score should be used to disqualify patients as too sick for LDLT. This uncertainty stems from the paucity of large studies evaluating the effect of MELD score on survival following LDLT. Therefore, the objective of our current study was to evaluate the association of MELD score and survival following LDLT in the U.S. population.

\section{Materials and Methods}

\section{Study population}

All adult patients (age 18 years and older) who underwent LDLT in the U.S. from 1995 to 2012 were evaluated using data from the United Network for Organ Sharing/Organ Procurement Transplant Network (UNOS/OPTN) registry. MELD score at the time of LDLT was used. Recipient and donor demographics were analyzed and included age at time of LDLT, sex, and race/ethnicity. Additional clinical characteristics for recipients, including body mass index (BMI), obesity, hepatitis C virus (HCV) infection, hepatocellular carcinoma (HCC), ascites, hepatic encephalopathy, and diabetes mellitus (DM) were analyzed. Data on graft-recipient weight ratio (GRWR) and graft weight/estimated standard liver weight (GW/ESLW) were not available. 


\section{Statistical analysis}

Clinical and demographic characteristics among LDLT recipients and donors were stratified into three MELD score categories: MELD < 15, MELD 15-19, and MELD 20-24. Categorical variables were presented as proportions and frequencies. Continuous variables were presented as mean \pm standard deviation. Comparisons among groups used $\chi^{2}$ testing for categorical variables and analysis of variance for continuous variables. Overall patient survival following LDLT was analyzed using the Kaplan-Meier method and log-rank testing for equality of survivor functions. Post-LDLT survival was stratified using the previously defined three MELD score categories. Multivariate Cox proportional hazards models were utilized to determine independent predictors of survival following LDLT. Forward stepwise regression methods included variables that were biologically important (e.g. age, sex), and those that demonstrated significant associations in the univariate models $(p<0.10)$. The final multivariate model was adjusted for age, sex, race/ethnicity, BMI, obesity, HCV infection, HCC, ascites, hepatic encephalopathy, and DM. Statistical significance was met with a two-tailed $p$ value $<0.05$. All statistical analyses were performed using the Stata statistical package (version 10; StataCorp, TX, USA).

Results

\section{Overview}

From 1995 to 2012, 2,258 patients underwent LDLT in the U.S., including 1,210 patients with MELD $<15$ (53.6\%), 732 with MELD 15-19 (32.4\%), and 316 with MELD 20-24 $(14.0 \%)$. Fig. 1 depicts the total number of LDLTS in the U.S. stratified by MELD score categories. There has been a general decline in the number of LDLTs occurring in the U.S. over the past decade (Fig. 1). The MELD < 15 cohort represents the greatest proportion of LDLT recipients, and each higher MELD cohort, namely MELD 15-19 and MELD 20-24, represent progressively lower proportions of LDLT recipients. Fig. 2 depicts the median MELD score of patients who underwent LDLT by year. Although there has been a general decline in the total number of LDLTs, the median MELD score shows a non-significant upward trend over the past decade (Fig. 2).

\section{Clinical and demographic characteristics}

Recipient and donor age, sex, BMI, obesity, HCV, and DM were similar among the three MELD categories (Table 1 ). Compared to patients with MELD $<15(63.0 \%)$, there was a significantly greater prevalence of ascites among those with MELD 15-19 $(79.8 \%)$ and MELD $20-24(82.0 \%)(p<0.001)$. The same prevalence trends were seen for hepatic encephalopathy: compared with MELD < 15 (50.4\%), MELD 15-19 (60.5\% vs. $50.4 \%, p<0.001)$ and MELD $20-24$ (63.9\% vs. $50.4 \%$, $p<0.001)$. Interestingly, rates of HCC were significantly lower among the MELD 15-19 (6.4\%) category compared to the MELD < $15(12.3 \%)$ and MELD 20-24 (12.0\%) categories $(p<0.001)$ (Table 1$)$. It should be noted that the biological MELD in patients with HCC may be irrelevant as HCC patients are listed with MELD exception.

\section{Survival following LDLT}

Compared to patients with MELD < 15, overall 5-year survival following LDLT was similar among patients with MELD 15-19 ( $80.9 \%$ vs. $80.3 \%, p=0.77)$ and MELD $20-24$ (81.2\% vs.

\section{Living Donor Liver Transplatations in the U.S.}

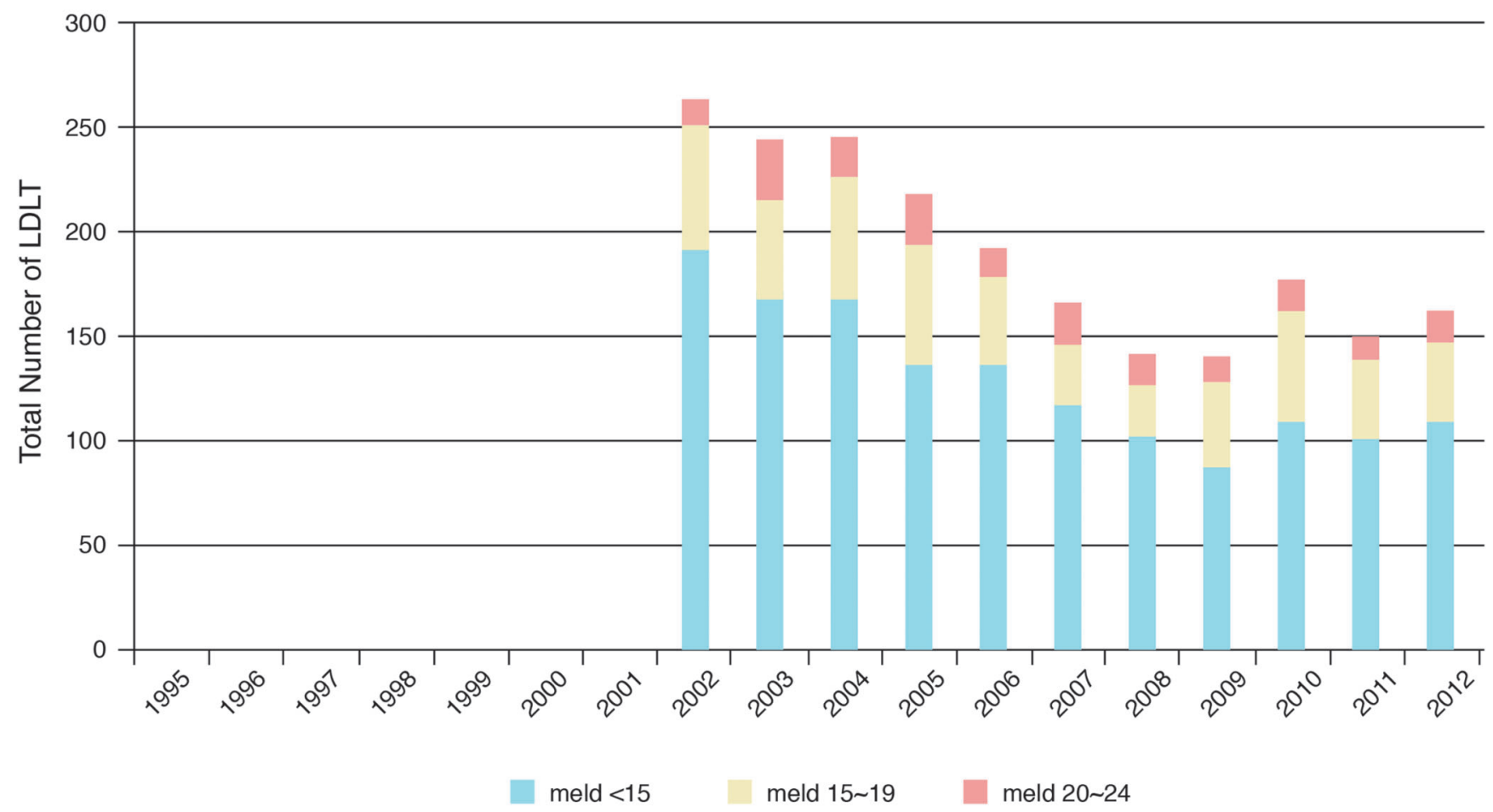

Fig. 1. Annual U.S. living donor liver transplantations by MELD score categories. 
Perumpail R.B. et al: Living donor liver transplantation

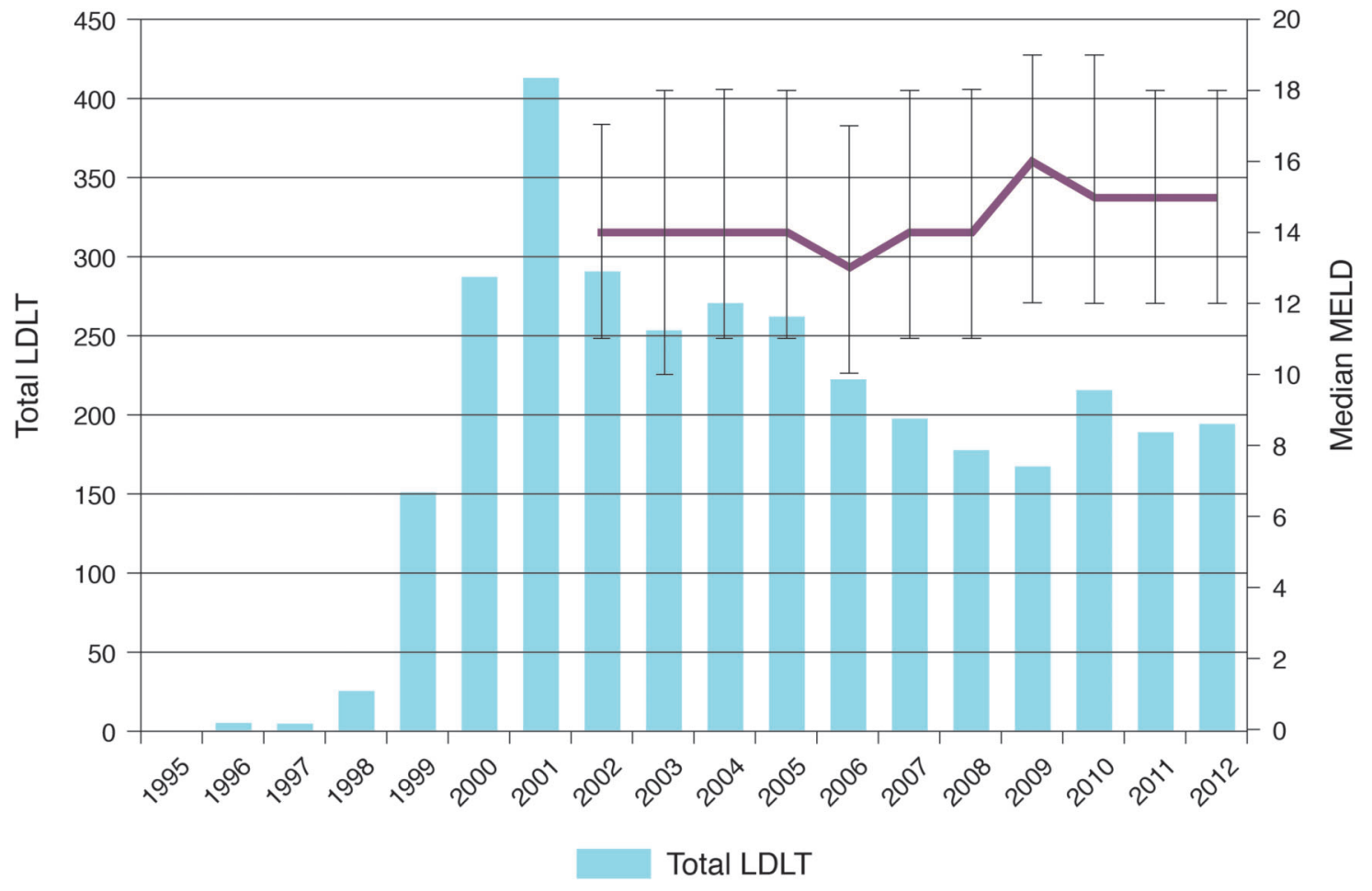

Fig. 2. Median MELD of living donor liver transplantation by year in U.S.

$80.3 \%, p=0.73$ ) (Figs. 3 and 4 ). Using multivariate Cox proportional hazards models, the independent impact of MELD score categories on survival following LDLT was evaluated (Table 2). When compared to patients with MELD $<15$, there was no significant difference in long-term post-LDLT survival among those with MELD 15-19 (HR: 1.11, 95\%
CI: $0.85-1.45, p=0.45)$ and a non-significant trend towards lower survival in patients with MELD 20-24 (HR: $1.28,95 \%$ CI: $0.91-1.81, p=0.16)$. The presence of HCC at the time of LDLT was a significant independent predictor of lower postLDLT survival (HR: $1.81,95 \% \mathrm{CI}: 1.25-2.63, p<0.01$ ). HCV (HR: 1.42, 95\% CI: $1.10-1.83, p<0.01$ ) and DM

Table 1. Liver donor liver transplantations in the U.S., 1995-2012

\begin{tabular}{|c|c|c|c|c|c|c|c|}
\hline & $\begin{array}{l}\text { MELD }<15 \\
(n=1,210)\end{array}$ & $n$ & $\begin{array}{l}\text { MELD 15-19 } \\
(n=732)\end{array}$ & $n$ & $\begin{array}{l}\text { MELD 20-24 } \\
(n=316)\end{array}$ & $n$ & $p$-Value \\
\hline Male & $54.7 \%$ & 662 & $59.8 \%$ & 438 & $57.6 \%$ & 182 & 0.08 \\
\hline Age, mean \pm SD & $51.9 \pm 11.4$ & & $51.6 \pm 11.3$ & & $51.6 \pm 11.9$ & & 0.80 \\
\hline BMI, mean \pm SD & $26.5 \pm 5.0$ & & $26.96 \pm 4.9$ & & $26.9 \pm 5.2$ & & 0.39 \\
\hline Obesity & $24.1 \%$ & 292 & $20.6 \%$ & 151 & $25.6 \%$ & 81 & 0.11 \\
\hline Race/ethnicity & & & & & & & 0.02 \\
\hline Non-Hispanic white & $85.2 \%$ & 1,024 & $81.4 \%$ & 592 & $81.6 \%$ & 257 & \\
\hline Black & $3.1 \%$ & 37 & $4.0 \%$ & 29 & $5.7 \%$ & 18 & \\
\hline Hispanic & $8.6 \%$ & 103 & $12.2 \%$ & 89 & $11.1 \%$ & 35 & \\
\hline Asian & $3.2 \%$ & 38 & $2.3 \%$ & 17 & $1.6 \%$ & 5 & \\
\hline Hepatitis C & $39.5 \%$ & 351 & $41.2 \%$ & 252 & $32.4 \%$ & 84 & 0.05 \\
\hline Hepatocellular carcinoma & $12.3 \%$ & 149 & $6.4 \%$ & 47 & $12.0 \%$ & 3.8 & $<0.001$ \\
\hline Ascites & $63.0 \%$ & 762 & $79.8 \%$ & 584 & $82.0 \%$ & 259 & $<0.001$ \\
\hline Hepatic encephalopathy & $50.4 \%$ & 610 & $60.5 \%$ & 443 & $63.9 \%$ & 202 & $<0.001$ \\
\hline MELD, mean \pm SD & $10.7 \pm 2.5$ & & $16.8 \pm 1.4$ & & $21.7 \pm 1.3$ & & $<0.001$ \\
\hline Diabetes & $12.8 \%$ & 137 & $13.2 \%$ & 87 & $12.4 \%$ & 37 & 0.95 \\
\hline
\end{tabular}


Post Transplantation Survival Living Donor Liver Transplantation

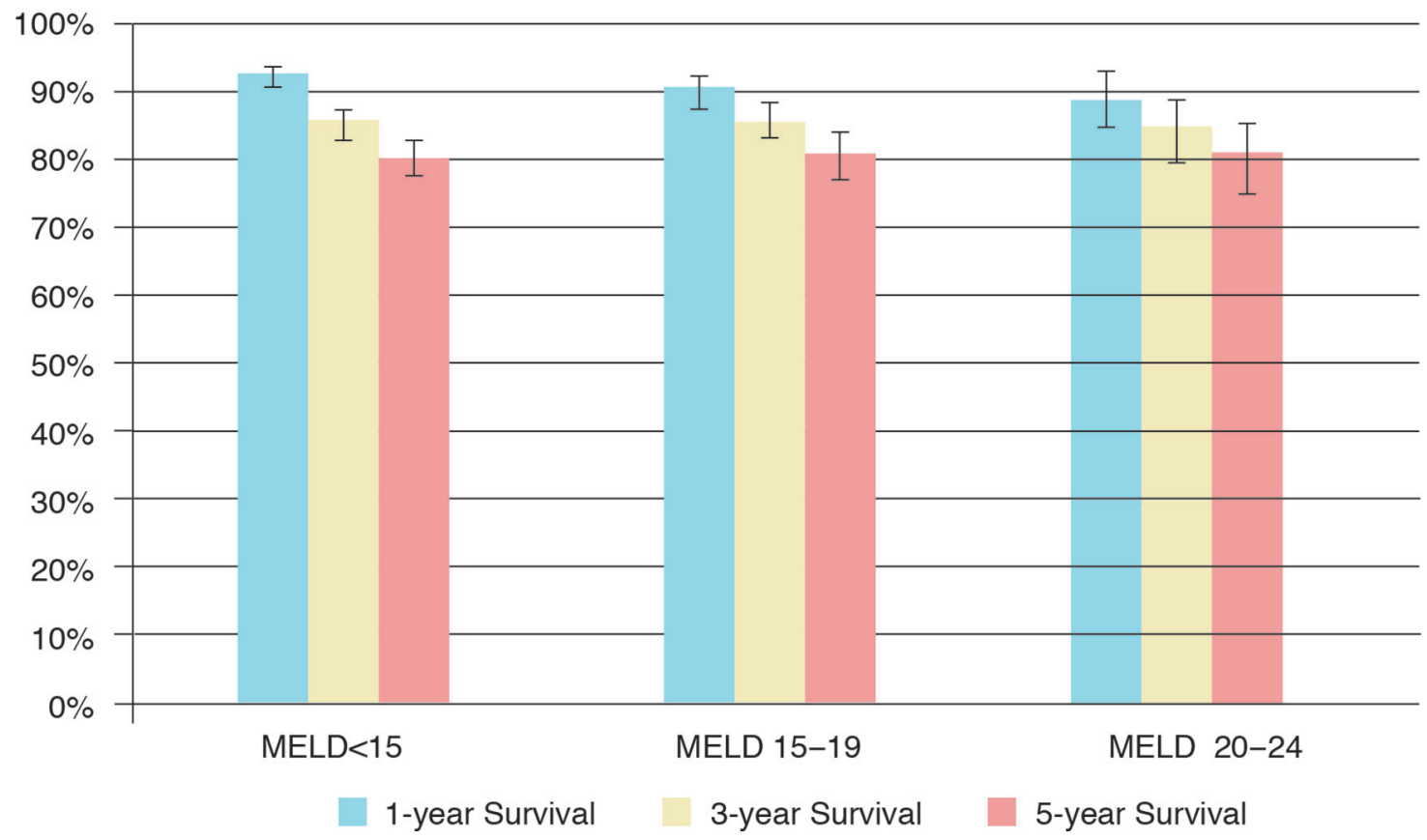

Fig. 3. Compared to patients with MELD <15, overall 1-year 3-year, and 5-year survival following LDLT were similar among patients with MELD 15-19 and MELD 20-24.

(HR: $1.52,95 \% \mathrm{CI}: 1.09-2.11, p<0.02)$ were also associated with lower survival following LDLT (Table 2).

\section{Discussion}

In the current study of adult LDLT recipients in the U.S. from 1995 to 2012, higher MELD scores were not associated with significantly lower survival following LDLT among the adult patients with MELD $<25$. Even after adjusting for potentially confounding variables in a multivariate model, including age, HCV, HCC and DM, higher MELD scores at the time of LDLT failed to demonstrate a negative impact on post-LDLT survival. However, advanced age, HCV, HCC, and DM were independently associated with lower survival following LDLT.

The paucity of data in the UNOS/OPTN database regarding LDLT recipients with MELD $\geq 25$ highlights the reservations that U.S. transplant centers have regarding offering LDLT to

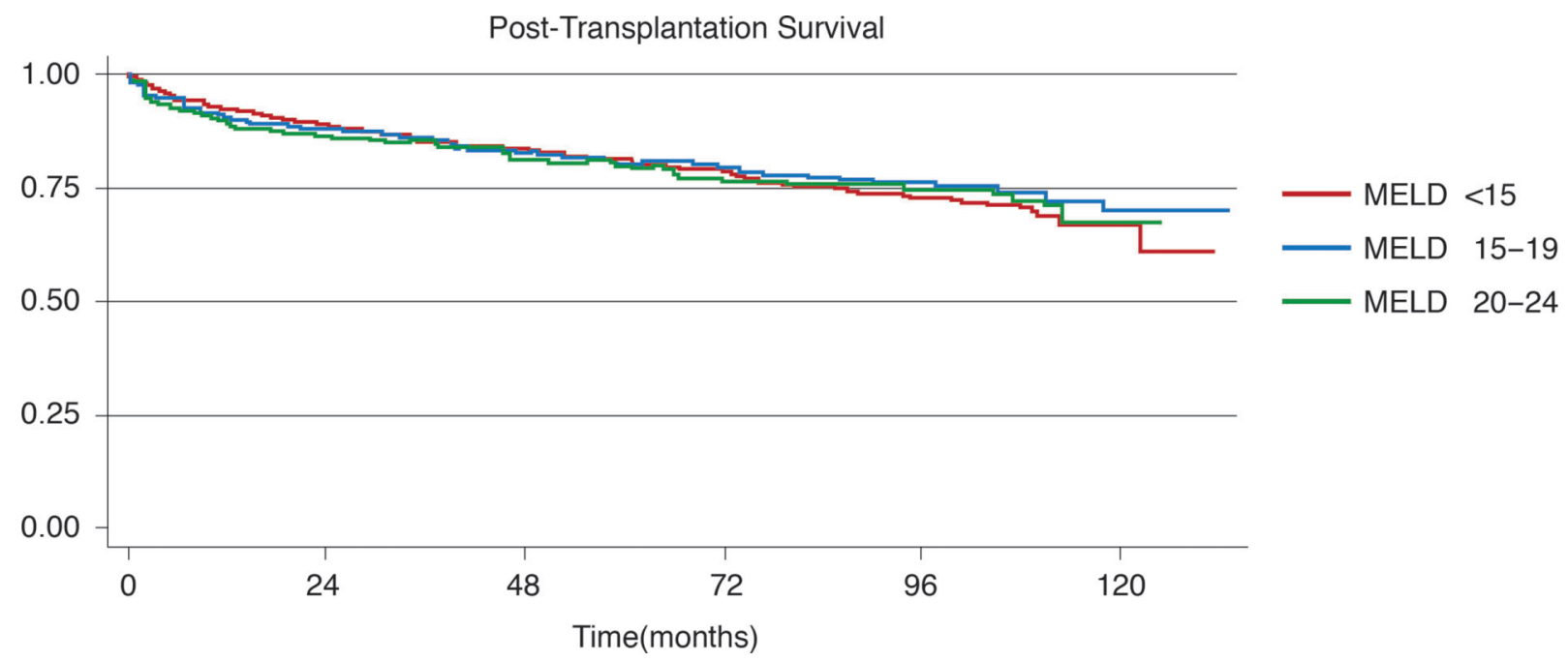

Fig. 4. Kaplan-Meier curves demonstrating that long-term survival following LDLT is similar among patients with MELD < 15, MELD 15-19, and MELD 20-24. 
Perumpail R.B. et al: Living donor liver transplantation

Table 2. Predictors of post-transplantation survival among living donor liver transplantations in U.S.

\begin{tabular}{|c|c|c|c|c|c|c|}
\hline & \multicolumn{3}{|c|}{ Univariate } & \multicolumn{3}{|c|}{ Multivariate } \\
\hline & $\mathrm{HR}$ & $95 \% \mathrm{CI}$ & $p$-Value & $\mathrm{HR}$ & $95 \% \mathrm{CI}$ & $p$-Value \\
\hline \multicolumn{7}{|l|}{ MELD Score } \\
\hline$<15$ & 1.00 & Reference & - & 1.00 & Reference & - \\
\hline $15-19$ & 0.96 & $0.77-1.19$ & 0.68 & 1.11 & $0.85-1.45$ & 0.45 \\
\hline $20-24$ & 1.01 & $0.76-1.36$ & 0.93 & 1.28 & $0.91-1.81$ & 0.16 \\
\hline Male (vs. female) & 1.15 & $1.00-1.33$ & 0.05 & 0.94 & $0.73-1.21$ & 0.63 \\
\hline Age & 1.03 & $1.02-1.04$ & $<0.001$ & 1.03 & $1.02-1.04$ & $<0.001$ \\
\hline Obesity & 1.21 & $1.03-1.43$ & $<0.01$ & 1.08 & $0.82-1.43$ & 0.57 \\
\hline \multicolumn{7}{|l|}{ Race/ethnicity } \\
\hline Non-Hispanic white & 1.00 & Reference & - & 1.00 & Reference & - \\
\hline Black & 1.40 & $1.01-1.94$ & $<0.05$ & 0.87 & $0.47-1.60$ & 0.64 \\
\hline Hispanic & 0.87 & $0.69-1.10$ & 0.24 & 0.84 & $0.56-1.25$ & 0.39 \\
\hline Asian & 0.98 & $0.65-1.48$ & 0.93 & 0.61 & $0.27-1.39$ & 0.24 \\
\hline Hepatitis C & 1.38 & $1.18-1.63$ & $<0.001$ & 1.42 & $1.10-1.83$ & $<0.01$ \\
\hline Hepatocellular carcinoma & 1.69 & $1.35-2.12$ & $<0.001$ & 1.81 & $1.25-2.63$ & $<0.01$ \\
\hline Ascites & 1.17 & $0.95-1.43$ & 0.15 & - & - & - \\
\hline Hepatic encephalopathy & 1.31 & $1.08-1.58$ & $<0.01$ & 1.27 & $0.97-1.65$ & 0.08 \\
\hline Diabetes & 1.48 & $1.12-1.96$ & $<0.01$ & 1.52 & $1.09-2.11$ & $<0.02$ \\
\hline
\end{tabular}

the sickest patients with end-stage liver disease. Moreover, the MELD $<15$ cohort represented the greatest proportion of LDLT recipients and each higher MELD cohort, namely MELD 15-19 and MELD 20-24, represented progressively lower proportions of LDLT recipients; this suggests that higher MELD scores are deterring hepatologists and liver transplant surgeons from offering this therapeutic option to sicker patients. This notion is corroborated by Samstein et al. ${ }^{2}$ who demonstrated a significant difference in MELD at transplant between DDLT and LDLT (mean, 20.3 vs. 15.2) among 1,036 liver transplant recipients. ${ }^{3}$ Analysis of U.S. registry data by Hoehn et al. ${ }^{4}$ demonstrated a median MELD score of 15 among LDLTs compared to 19 among DDLTs. Between 2007 and 2012, only 14 LDLTs were performed among patients with MELD 28-34 and 3 among patients with MELD 35-40. ${ }^{4}$

The number of LDLTs in the U.S. peaked in 2001, when 506 were performed. ${ }^{7}$ Subsequently, media attention surrounding the donor death of a New York man who donated the right lobe of his liver to his brother spurred a decline in LDLTs performed, down to 353 in 2002. ${ }^{7}$ The decline persisted over the decade to follow. However, a recent study by Muzaale et al. ${ }^{8}$ reveals that the risk of early death, defined as within 3 months of LDLT, from live liver donation in the U.S. is only 1.7 per 1000 donors. Moreover, these investigators found that overall survival among live liver donors did not differ from that of healthy, matched individuals from the National Health and Nutrition Examination Survey (NHANES) over a mean follow-up of 7.6 years. ${ }^{8}$ This finding is akin to previous data demonstrating that long-term overall survival among live kidney donors did not differ from a comparably matched cohort from NHANES, which is significant because live kidney donation is generally regarded as safe and has become widespread in the U.S. ${ }^{9}$ Furthermore, long-term prospective follow-up of 372 live liver donors reveals that most maintain above average health-related quality of life up to 11 years following LDLT. ${ }^{10}$ Given growing evidence of donor safety after live donation, LDLT may be ready for more widespread availability for patients with end-stage liver disease in the U.S.

There is vast international experience with LDLT, particularly in East Asian nations, and specifically in Japan and South Korea, where cultural factors significantly limit adoption of deceased organ donation. In the U.S., the multiinstitutional Adult-to-Adult Living Donor Liver Transplantation Study (A2ALL) prospectively involved 9 major transplant centers and demonstrated overall 1-year and 3-year patient survivals of $94 \%$ and $78 \%$, respectively. ${ }^{11}$ Goldberg et al. ${ }^{12}$ demonstrated that overall patient survival following LDLT is equivalent, if not superior, to DDLT when performed at experienced centers based on UNOS/OPTN registry data.

Data assessing the impact of MELD score on survival following LDLT are lacking. Existing literature is from foreign centers, limited in sample size, or dated. Single center data for 335 patients who underwent LDLT in Japan suggested that MELD $\geq 20$ was an independent predictor of lower graft survival (HR: $2.9,95 \% \mathrm{CI}: 1.6-5.2, p<0.01$ ), but these investigators did not evaluate the association of MELD with overall patient survival. ${ }^{13}$ Further analysis from the same center revealed no difference in graft survival following LDLT between 46 patients with MELD $\geq 25$ and 311 patients with MELD $<25 .{ }^{14}$ Data from a large Korean university hospital analyzing 167 LDLTs performed between 1999 and 2005 demonstrated that MELD > 25 did not predict 1-year patient survival. Similarly, single center data from a Canadian center analyzed 271 patients who underwent LDLT between 2002 to 2008 and reported comparable 1-year, 3-year and 5-year graft and patient survival in MELD $<25$ and MELD $\geq 25$ groups. ${ }^{15}$ In a single center U.S. study, Hayashi et al. ${ }^{16}$ evaluated the association of MELD scores at time of transplant 
with 1-year post-LDLT graft and patient survival; they noted that MELD predicts neither overall graft nor patient survival following LDLT.

Overall, the existing literature fails to provide a consensus on the U.S. experience in LDLT. Asian data regarding the adoption and success of LDLT may not be extrapolated to the U.S. population due to higher prevalence of chronic hepatitis B and HCC in Asia. In addition, a larger volume of LDLT in Asia may be associated with differences in surgical experience and outcomes versus the U.S. population. In light of this, the findings of our study provide valuable insight into outcomes following LDLT in the U.S. and suggest that LDLT can be offered to sicker patients with higher MELD scores without compromising survival outcomes.

The current study uses population-based data that includes all adult LDLTs performed in the U.S. from 1995 to 2012 . The comprehensive nature of the cohort improves consistency of comparisons among geographic areas and minimizes the potential for selection bias, improving overall generalizability. However, our study is limited by factors inherent in registrybased research and lack of granular data, which may be significant; this would need to be studied in future analysis. Our retrospective study design limited the ability to evaluate the accuracy of the information captured. Furthermore, our study reveals that LDLTs are rarely performed on patients with $M E L D \geq 25$, which precluded us from including this cohort in our analysis due to the limited sample size. Despite these limitations, the utilization of large population-based data stratified by MELD categories adds considerable strength and generalizability to our findings.

In conclusion, our large population-based study of U.S. adult LDLT recipients demonstrates that survival following LDLT is not affected by MELD scores up to 25 . LDLT is underutilized in patients with MELD score 20 and higher. Our study findings suggest that LDLT can be offered as a therapeutic option to patients with chronic liver disease and MELD score between 20-24. Indeed, we are not suggesting a MELD cutoff of 25 as an exclusion criterion for LDLT. Rather, we want to highlight the current lack of LDLT being performed in our sickest patients and would recommend that a patient-specific approach be taken to assess candidacy in this patient population. Large prospective studies are warranted to investigate outcomes of LDLT in patients with MELD $\geq 25$.

\section{Conflict of interest}

None

\section{Author contributions}

Study concept and design (RBP, ERY, GC, LH, MD, WCC, CAB, ZMY, RJW, AA), acquisition of data (RBP), analysis and interpretation of data (RBP, ERY, GC, LH, MD, WCC, CAB, ZMY, RJW, $A A)$, drafting of the initial and final manuscript (RBP, ERY, GC, LH, MD, WCC, CAB, ZMY, RJW, AA), critical revision of the manuscript (ERY, GC, LH, MD, WCC, CAB, ZMY, RJW, $A A$ ), and study supervision (AA).

\section{References}

[1] Kim WR, Lake JR, Smith JM, Skeans MA, Schladt DP, Edwards EB, et al. OPTN/SRTR 2013 Annual Data Report: liver. Am J Transplant 2015;15 Suppl 2:1-28. doi: 10.1111/ajt.13197.

[2] Samstein B, Smith A, Freise C, Zimmerman M, Baker T, Olthoff K, et al. Posttransplant complications in recipients of deceased and living donor liver transplant [Abstract]. Am J Transplant 2014;14:2.

[3] Mueller TF, Oberkofler CE, Clavien PA. What's hot, what's new at WTC-clinical science. Am J Transplant 2015;15:327-332. doi: 10.1111/ajt.13081.

[4] Hoehn RS, Wilson GC, Wima K, Hohmann SF, Midura EF, Woodle ES, et al. Comparing living donor and deceased donor liver transplantation: A matched national analysis from 2007 to 2012. Liver Transpl 2014;20:1347-1355. doi : $10.1002 /$ It. 23956.

[5] Kamath PS, Wiesner RH, Malinchoc M, Kremers W, Therneau TM, Kosberg CL, et al. A model to predict survival in patients with end-stage liver disease. Hepatology 2001;33:464-470. doi: 10.1053/jhep.2001.22172.

[6] Wiesner R, Edwards E, Freeman R, Harper A, Kim R, Kamath P, et al. Model for end-stage liver disease (MELD) and allocation of donor livers. Gastroenterology 2003;124:91-96. doi: 10.1053/gast.2003.50016.

[7] Vastag B. Living-donor transplants reexamined: experts cite growing concerns about safety of donors. JAMA 2003;290:181-182. doi: 10.1001/ jama.290.2.181.

[8] Muzaale AD, Dagher NN, Montgomery RA, Taranto SE, McBride MA, Segev DL. Estimates of early death, acute liver failure, and long-term mortality among live liver donors. Gastroenterology 2012;142:273-280. doi: $10.1053 / \mathrm{j}$. gastro.2011.11.015.

[9] Segev DL, Muzaale AD, Caffo BS, Mehta SH, Singer AL, Taranto SE, et al. Perioperative mortality and long-term survival following live kidney donation. JAMA 2010;303:959-966. doi: 10.1001/jama.2010.237.

[10] Ladner DP, Dew MA, Forney S, Gillespie BW, Brown RS Jr, Merion RM, et al. Long-term quality of life after liver donation in the adult to adult living donor liver transplantation cohort study (A2ALL). J Hepatol 2015;62:346-353. doi 10.1016/j.jhep.2014.08.043.

[11] Berg CL, Gillespie BW, Merion RM, Brown RS Jr, Abecassis MM, Trotter JF, et al. Improvement in the survival associated with adult-to-adult living donor liver transplantation. Gastroenterology 2007;133:1806-1813. doi: 10.1053/ j.gastro.2007.09.004

[12] Goldberg DS, French B, Abt PL, Olthoff K, Shaked A. Superior survival using living donors and donor-recipient matching using a novel living donor risk index. Hepatology 2014;60:1717-1726. doi: 10.1002/hep.27307.

[13] Ikegami T, Imai D, Wang $H$, Yoshizumi T, Yamashita $Y$, Ninomiya M, et al. D-MELD as a predictor of early graft mortality in adult-to-adult living-donor liver transplantation. Transplantation 2014;97:457-462. doi: 10.1097/01. TP.0000435696.23525.d0.

[14] Ikegami T, Shirabe K, Yoshiya S, Yoshizumi T, Yamashita Y, Harimoto N, et al. A high MELD score, combined with the presence of hepatitis $C$, is associated with a poor prognosis in living donor liver transplantation. Surg Today 2014; 44:233-240. doi: 10.1007/s00595-013-0523-7.

[15] Selzner M, Kashfi A, Cattral MS, Selzner N, McGilvray ID, Greig PD, et al. Live donor liver transplantation in high MELD score recipients. Ann Surg 2010; 251:153-157. doi: 10.1097/SLA.0b013e3181bc9c6a.

[16] Hayashi PH, Forman L, Steinberg T, Bak T, Wachs M, Kugelmas M, et al. Model for end-stage liver disease score does not predict patient or graft survival in living donor liver transplant recipients. Liver Transpl 2003;9:737-740. doi: $10.1053 /$ jlts.2003.50122. 